\title{
Robust $\mathscr{H}_{\infty}$ Dynamic Output Feedback Control Synthesis with Pole Placement Constraints for Offshore Wind Turbine Systems
}

\author{
Tore Bakka and Hamid Reza Karimi \\ Department of Engineering, Faculty of Engineering and Science, University of Agder, \\ 4879 Grimstad, Norway \\ Correspondence should be addressed to Hamid Reza Karimi, hamid.r.karimi@uia.no
}

Received 7 June 2012; Accepted 21 September 2012

Academic Editor: Zidong Wang

Copyright (C) 2012 T. Bakka and H. R. Karimi. This is an open access article distributed under the Creative Commons Attribution License, which permits unrestricted use, distribution, and reproduction in any medium, provided the original work is properly cited.

\begin{abstract}
The problem of robust $\mathscr{H}_{\infty}$ dynamic output feedback control design with pole placement constraints is studied for a linear parameter-varying model of a floating wind turbine. A nonlinear model is obtained and linearized using the FAST software developed for wind turbines. The main contributions of this paper are threefold. Firstly, a family of linear models are represented based on an affine parameter-varying model structure for a wind turbine system. Secondly, the bounded parameter-varying parameters are removed using upper bounded inequalities in the control design process. Thirdly, the control problem is formulated in terms of linear matrix inequalities (LMIs). The simulation results show a comparison between controller design based on a constant linear model and a controller design for the linear parameter-varying model. The results show the effectiveness of our proposed design technique.
\end{abstract}

\section{Introduction}

Wind energy is nowadays one of the fastest growing renewable industries. As a consequence of the oil crises in the early 1970s and a general interest of renewable energy, the wind energy sector has had a tremendous growth over the last decades. With Europe leading the global market, the turbine capacity has had an annual growth rate of up to $30 \%$ [1].

Wind turbines are complex mechanical systems, and they are highly nonlinear due to the conversion of wind energy to mechanical torque. This makes the wind turbine a challenging task both to model and control. In literature, linear and nonlinear controllers have been extensively used for power regulation through the control of blade pitch angle (see, for instance, [2-14] and the references therein). More recently, the problem of gain scheduling and output feedback $\mathscr{H}_{\infty}$ control design for an offshore floating wind turbine was studied in $[15,16]$. Furthermore, a mixed $\mathscr{L}_{2} / \mathscr{L}_{\infty}$ control design was proposed for an offshore floating 


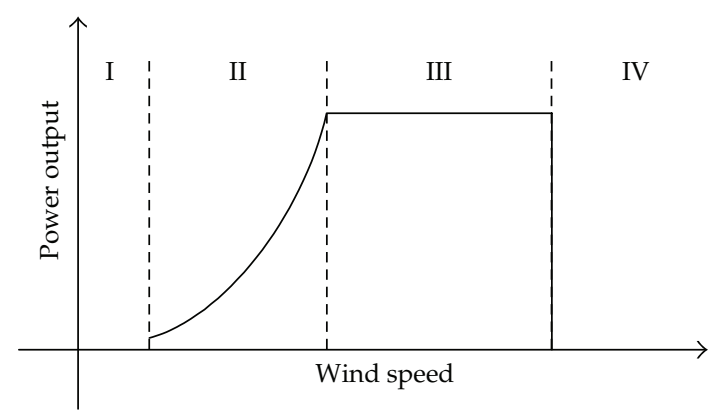

Figure 1: Operating region of a typical wind turbine.

wind turbine system investigated in [17]. However, the performance of these controllers is limited by the highly nonlinear characteristics of the wind turbine. These controllers are designed on the basis of one operating condition and therefor can only guarantee performance and stability at this point. By designing the controller on the basis of a linearparameter-varying (LPV) model, it is possible to overcome these limitations. So, in order to sustain the growth in the wind industry sector, design of advanced control methodologies is one research area where such improvements can be achieved. In recent years, several advanced wind turbine simulation softwares have emerged, such as HAWC2 [18], FAST [19], and Cp-Lambda [20]. In this paper we will use FAST interfaced with MATLAB for all the simulations. The operation region of a wind turbine is often divided into four regions (Figure 1).

In region I $\left(v<v_{\text {cut-in }}\right)$ the wind speed is lower than the cut-in wind speed and no power can be produced. In region II ( $\left.v_{\text {cut-in }} \leq v<v_{\text {rated }}\right)$ the pitch is usually kept constant while the generator torque is the controlling variable. In region III $\left(v_{\text {rated }} \leq v<v_{\text {cut-out }}\right)$ the main concern is to keep the rated power and to limit loads on critical parts of the structure by pitching the blades. In region IV ( $\left.v \leq v_{\text {cut-out }}\right)$ the wind speed is too high, and the turbine is shut down. In this paper we will focus on the above rated wind speed scenario, that is, region III.

This paper makes three specific contributions. First, it suggests a family of linear models for a wind turbine system based on an affine parameter-varying model structure. Second, robust stabilization and disturbance attenuation of such parameter-varying models are investigated using $\mathscr{l}_{\infty}$ method such that the bounded parameter-varying parameters are removed using upper bounded inequalities in the control design procedure. Third, the control problem is formulated in terms of linear matrix inequalities (LMIs) and a dynamic output feedback controller is computed. Finally, the simulation results show that the obtained controller can achieve the robust stability and disturbance attenuation, simultaneously.

This paper is organized as follows. Section 2 describes the model under consideration and how to include the parameter-varying terms in the closed loop system. Section 3 is devoted to the control design technique. Simulation results are presented in Section 4. Finally, concluding remarks and suggestions to future works are discussed in Section 5.

The notations used throughout the paper are fairly standard. $I$ and 0 represent identity matrix and zero matrix; the superscript $T$ stands for matrix transposition; $\mathfrak{R}^{n}$ denotes the $n$-dimensional Euclidean space; $\mathfrak{R}^{n \times m}$ is the set of all real $m$ by $n$ matrices. $\|\cdot\|$ refers to the Euclidean vector norm or the induced matrix 2-norm. diag $\{\cdots\}$ represents a block diagonal matrix. The operator $\operatorname{sym}(A)$ denotes $A+A^{T}$, and $\otimes$ denotes the Kronecker product. The notation $P>0$ means that $P$ is real symmetric and positive definite; the symbol $*$ denotes 
the elements below the main diagonal of a symmetric block matrix. Finally given a signal $x(z),\|x(z)\|_{2}$ denotes the $L_{2}$ norm of $x(z)$, that is, $\|x(z)\|_{2}^{2}=\int_{0}^{\infty} x^{T}(z) x(z) d t$.

\section{Wind Turbine Model}

The wind turbine model is obtained from the wind turbine simulation software FAST [19]. The simulation model is an upscaled version of Statoil's Hywind 2.3 (MW) turbine, which is located off the Norwegian west coast. This upscaled version is also a floating turbine and has the capacity 5 (MW). For specifications, see [21].

FAST provides a fully nonlinear wind turbine model with up to 24 degrees of freedom (DOF). For the controller design, we need a linear model and we want the linear model to be as simple as possible. All the DOFs available cannot be included, so we choose the ones we think will represent the most important dynamics. Linearization routines are available in the FAST package. The model is now linearized at each desired azimuth angle. We find this angle in the plane of rotor rotation. One linear model at each 10th angle is obtained, that is, the total amount of 36 models are obtained. The models is of the following standard state space form:

$$
\begin{gathered}
\dot{x}=A_{i} x+B_{i} u, \\
y=C_{i} x, \quad i=1,2, \ldots, 36
\end{gathered}
$$

where $x$ is the state vector with dimensions $\mathcal{R}^{n \times 1}, u$ is the control signal with dimensions $\mathcal{R}^{p \times 1}, y$ is the model outputs with dimensions $\mathcal{R}^{m \times 1}$, and $A, B, C$ are the system matrices with dimensions $\mathcal{R}^{n \times n}, \mathcal{R}^{n \times p}, \mathcal{R}^{m \times n}$, and $\mathcal{R}^{m \times p}$, respectively. The states in this linear model are tower fore-aft displacement $\left(x_{1}\right)$, generator position $\left(x_{2}\right)$, rotor position $\left(x_{3}\right)$, and the last three states are the first derivative of $x_{1-3}$. The model input $u$, which will eventually be calculated by the controller, is the blade pitch angle. The model outputs in $y$ are tower fore-aft displacement, generator speed, and rotor speed.

A common way to simplify these models is to take the average of all the 36 models and use this as basis for the controller design. By doing this simplification, important information is easily lost. This is why in this paper we will try to do the controller design based on a model representation which tries to include as much as possible of the information in the 36 models. The matrices $A$ and $B$ are behaving in a periodic way, and the matrix values depend on the rotor azimuth angle. Several things are the cause of this periodic behavior, that is, aerodynamic loads, tower shadow, gravitational loads, and deflections of the tower due to thrust loading. The matrix associated with the output $y$ is not varying, since this $C$-matrix only handles the measurements. In (2.2) we define the varying matrices in an affine way, and $A(z)$ and $B(z)$ vary in a continuous manner:

$$
\begin{aligned}
& A(z)=A_{n}+\Delta A(z), \\
& B(z)=B_{2 n}+\Delta B(z),
\end{aligned}
$$

where $A_{n}$ and $B_{2 n}$ are the nominal plant matrices, $\Delta A(z)$ and $\Delta B(z)$ contributes with the varying terms, and $z$ represents the rotor azimuth angle. We are looking to represent the parameter-varying terms in this way: $\Delta A(z)=F \Delta(z) E$, and a similar expression for $\Delta B(z)$. After analyzing the 36 models we find appropriate matrices $F$ and $E$, but we also find out 
that more than one scheduling parameter is needed. The periodic matrices $A(z)$ and $B(z)$ can now be represented in a continuous way with the use of sine and cosine functions. The parameter-varying terms in (2.2) are defined in the following way:

$$
\begin{aligned}
& \Delta A(z)=\sum_{i=1}^{2} \sum_{j=1}^{2} F_{i} \Delta_{j}(z) E_{j i a}, \\
& \Delta B(z)=\sum_{i=1}^{2} \sum_{j=1}^{2} F_{i} \Delta_{j}(z) E_{j i b},
\end{aligned}
$$

where the vectors $F$ and $E$ have appropriate dimensions, and the scheduling variables $\Delta_{1}(z)$ and $\Delta_{2}(z)$ are found to be $\sin (\omega t)$, and $\cos (\omega t)$ respectively. A plot which shows what the different parameters are in the original matrices $A_{1, \ldots, 36}$ and $B_{1, \ldots, 36}$ and in the new representation $A_{n}+\Delta A(z)$ and $B_{2 n}+\Delta B(z)$ is found in the appendix.

\section{Control Design}

The purpose of $\mathscr{H}_{\infty}$ control is to minimize the effect of disturbances on the controlled output. The control design is formulated in terms of LMIs. After manipulating the linear model obtained from FAST, we end up with a state space system with parameter-varying $A$ and $B$ matrices. This model is more accurate than if we just took the average of all the 36 models. By using a LPV model of the system we are able to catch some of the dynamics that are lost under the linearization. The challenge is now to incorporate these additional terms into the control design.

These robust control designs mostly deal with frequency domain aspects of the closed loop system, but it is well known that the location of the closed loop poles play a large role in the transient behavior of the controlled system. By adding pole placement to the list of constraints we can prevent large poles and end up with a system which can respond in a realistic way. The controller we are searching for will try to keep the generator speed at its rated value while mitigating oscillations in the drive train and in the tower.

The LMIs for the control design are solved using YALMIP [22] interfaced with MATLAB, and we are using the solver SeDuMi. This solver is searching for two positive definite matrices $X$ and $Y$ which stabilizes the system. If these matrices exist, we can calculate the controller. The next sections present how to obtain the LMIs for the controller design and also how to incorporate the parameter-varying part of the state space system.

\subsection{System Representation}

Figure 2 shows the output feedback control scheme, where $P(s)$ is the generalized plant and $K(s)$ is the controller. The two blocks represent in the equations (3.1) and (3.2). $P(s)$ includes the wind turbine model and the signals of interest:

$$
\begin{aligned}
& \dot{x}=A x+B_{1} w+B_{2} u \\
& z_{\infty}=C_{1 i} x+D_{1 i} w+D_{2 i} u \\
& y=C_{2} x+D_{21} w
\end{aligned}
$$




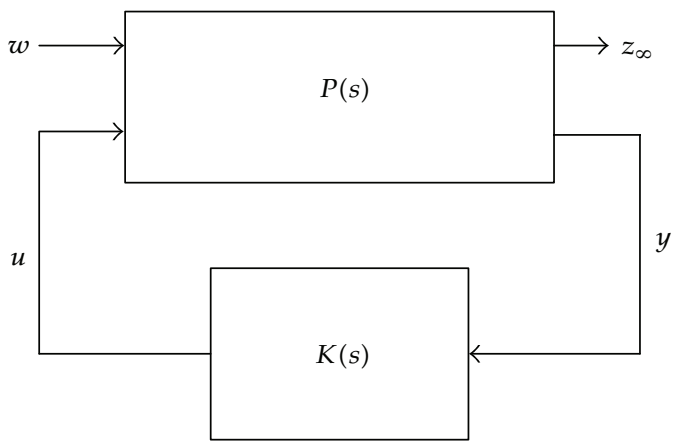

Figure 2: Output feedback block diagram.

where $A, B_{2}$, and $C_{2}$ represent the matrices from the standard state space form in (2.1). To include the parameter-varying matrices, $A$ is substituted with $A(z)$ and $B_{2}$ with $B(z)$. The other matrices are considered with appropriate dimensions. $u$ is the control input, $w$ is the disturbance signal, and $y$ is the measured output. The signal $z_{\infty}$ is the controlled output for $\mathscr{H}_{\infty}$ performance measure. For system (3.1), the dynamic output feedback, $u(s)=K(s) y(s)$, is of the following form:

$$
K(s)\left\{\begin{array}{l}
\dot{\zeta}=A_{k} \zeta+B_{k} y, \\
u=C_{k} \zeta+D_{k} y .
\end{array}\right.
$$

The closed loop system is given in (3.3) with the states $x_{\mathrm{cl}}=\left[\begin{array}{ll}x \zeta \\ \zeta\end{array}\right]^{T}$ :

$$
\begin{aligned}
& \dot{x}_{\mathrm{cl}}=A_{\mathrm{cl}} x+B_{\mathrm{cl}} w, \\
& z_{\infty}=C_{\mathrm{cl}} x+D_{\mathrm{cl}} w .
\end{aligned}
$$

The closed loop system is divided into two parts, one with constant state space matrices and one where the parameter-varying matrices are

$$
\begin{aligned}
\left(\begin{array}{c|c}
A_{\mathrm{cl}} & B_{\mathrm{cl}} \\
\hline C_{\mathrm{cl}} & D_{\mathrm{cl}}
\end{array}\right)= & \left(\begin{array}{c|c|c}
A_{\mathrm{cl} 1} & B_{\mathrm{cl} 1} \\
\hline C_{\mathrm{cl} 1} & D_{\mathrm{cl} 1}
\end{array}\right)+\left(\begin{array}{cc|c}
A_{\mathrm{cl} 2}(z) & B_{\mathrm{cl} 2}(z) \\
\hline 0 & 0
\end{array}\right) \\
& =\left(\begin{array}{cc|c}
A_{n}+B_{2 n} D_{k} C_{2} & B_{2 n} C_{k} & B_{1}+B_{2 n} D_{k} D_{21} \\
B_{k} C_{2} & A_{k} & B_{k} D_{21} \\
\hline C_{1 i}+D_{2 i} D_{k} C_{2} & D_{2 i} C_{k} & D_{1 i}+D_{2 i} D_{k} D_{21}
\end{array}\right) \\
& +\left(\begin{array}{cc|c}
\Delta A(z)+\Delta B(z) D_{k} C_{2} & \Delta B(z) C_{k} & \Delta B(z) D_{k} D_{21} \\
0 & 0 & 0 \\
\hline 0 & 0 & 0
\end{array}\right) .
\end{aligned}
$$




\section{2. $\mathscr{H}_{\infty}$ Control}

Because of the parameter-varying state space system we now get an additional term to the standard Bounded Real Lemma (BRL). This additional term is the second part of the summation in constraint (3.5). We want to make sure that the closed loop $\mathscr{H}_{\infty}$ norm of the closed loop transfer function does not exceed $\gamma$. This is true if and only if there exists a symmetric matrix $X$ such that

$$
\left(\begin{array}{ccc}
A_{\mathrm{cl} 1}^{T} X+X A_{\mathrm{cl} 1} & X B_{\mathrm{cl} 1} & C_{\mathrm{cl} 1}^{T} \\
* & -\gamma I & D_{\mathrm{cl} 1}^{T} \\
* & * & -\gamma I
\end{array}\right)+\left(\begin{array}{ccc}
A_{\mathrm{cl} 2}^{T}(z) X+X A_{\mathrm{cl} 2}(z) & X B_{\mathrm{cl} 2}(z) & 0 \\
* & 0 & 0 \\
* & * & 0
\end{array}\right)<0
$$

$$
X>0 .
$$

\subsection{Change of Variables}

Obviously, the $\mathscr{H}_{\infty}$ constraint (3.5) is not an LMI because of the nonlinear terms which occur when we close the loop. In order to transform these nonlinear terms into proper LMIs we need to do two things. First, we need to linearize them with the use of change of variables. Second, we need to remove the parameter-varying terms. The linearization part is not as straight forward as for the state feedback case, additional information about this can be found in [23].

The new Lyapunov matrix is partitioned in the following form:

$$
x=\left[\begin{array}{cc}
Y & N \\
N^{T} & \#
\end{array}\right], \quad X^{-1}=\left[\begin{array}{cc}
X & M \\
M^{T} & \#
\end{array}\right]
$$

where $X$ and $Y$ are symmetric matrices of dimension $n \times n$. It is not necessary to know the matrices noted as \#.

In addition, we define the following two matrices:

$$
\Pi_{1}=\left[\begin{array}{cc}
X & I \\
M^{T} & 0
\end{array}\right], \quad \Pi_{2}=\left[\begin{array}{cc}
I & Y \\
0 & N^{T}
\end{array}\right]
$$

that, as can be inferred from the identity $x x^{-1}=I$, satisfy

$$
x \Pi_{1}=\Pi_{2} .
$$


Then, the following change of controller variables are defined:

$$
\begin{gathered}
\widehat{A}=N A_{k} M^{T}+N B_{k} C_{2} X+Y B_{2 n} C_{k} M^{T}+Y\left(A_{n}+B_{2 n} D_{k} C_{2}\right) X, \\
\widehat{B}=N B_{k}+Y B_{2 n} D_{k}, \\
\widehat{C}=C_{k} M^{T}+D_{k} C_{2} X, \\
\widehat{D}=D_{k} .
\end{gathered}
$$

Now we are ready to convert our nonlinear matrix inequalities into LMIs. By performing congruence transformation with $\operatorname{diag}\left(\Pi_{1}, I, I\right)$ on the obtained inequality (3.5), we end up with following matrix inequality:

$$
\begin{aligned}
\Sigma_{1} & +\operatorname{sym}\left(G_{1} \Delta_{1}(z) H_{1}\right)+\operatorname{sym}\left(G_{2} \Delta_{1}(z) H_{1}\right) \\
& +\operatorname{sym}\left(G_{1} \Delta_{1}(z) H_{2}\right)+\operatorname{sym}\left(G_{2} \Delta_{1}(z) H_{2}\right) \\
& +\operatorname{sym}\left(G_{3} \Delta_{2}(z) H_{3}\right)+\operatorname{sym}\left(G_{4} \Delta_{2}(z) H_{3}\right) \\
& +\operatorname{sym}\left(G_{3} \Delta_{2}(z) H_{4}\right)+\operatorname{sym}\left(G_{4} \Delta_{2}(z) H_{4}\right)<0,
\end{aligned}
$$

where the matrix $\Sigma_{1}$ and the vectors $G_{i}$ and $H_{i}$ are defined in the appendix.

Lemma 3.1 (see [24]). Given $\Sigma=\Sigma^{T}, G, \Delta$, and $H$ of appropriate dimensions with $\Delta^{T} \Delta \leq I$, then the matrix inequality

$$
\Sigma+(G \Delta H)<0
$$

holds for all $\Sigma$ if and only if there exists a scalar $\epsilon>0$ such that

$$
\Sigma+\epsilon G G^{T}+\epsilon^{-1} H^{T} H<0
$$

By using Lemma 3.1 we are able to remove the parameter-varying parts $\Delta_{i}(z)$ in the matrix inequality (3.10). We end up with a new LMI which contains the constants $\epsilon_{1}$ and $\epsilon_{2}$ :

$$
\begin{aligned}
\Sigma_{1} & +2 \epsilon_{1} G_{1} G_{1}^{T}+2 \epsilon_{1}^{-1} H_{1}^{T} H_{1}+2 \epsilon_{1} G_{2} G_{2}^{T}+2 \epsilon_{1}^{-1} H_{2}^{T} H_{2} \\
& +2 \epsilon_{2} G_{3} G_{3}^{T}+2 \epsilon_{2}^{-1} H_{3}^{T} H_{3}+2 \epsilon_{2} G_{2} G_{4}^{T}+2 \epsilon_{2}^{-1} H_{4}^{T} H_{4}<0 .
\end{aligned}
$$

By using the Schur complement we can convert (3.13) into the following LMIs:

$$
\begin{aligned}
& \left(\begin{array}{cc}
\Sigma_{1} & \Sigma_{2} \\
* & \Sigma_{3}
\end{array}\right)<0 \\
& \left(\begin{array}{ll}
X & I \\
I & Y
\end{array}\right)>0
\end{aligned}
$$


where

$$
\begin{array}{r}
\Sigma_{2}=\left[\begin{array}{llllllll}
G_{1} & H_{1}^{T} & G_{2} & H_{2}^{T} & G_{3} & H_{3}^{T} & G_{4} & H_{4}^{T}
\end{array}\right] \\
\Sigma_{3}=\operatorname{diag}\left\{-\frac{1}{2} \epsilon_{1}^{-1},-\frac{1}{2} \epsilon_{1},-\frac{1}{2} \epsilon_{1}^{-1},-\frac{1}{2} \epsilon_{1},\right. \\
\left.-\frac{1}{2} \epsilon_{2}^{-1},-\frac{1}{2} \epsilon_{2},-\frac{1}{2} \epsilon_{2}^{-1},-\frac{1}{2} \epsilon_{2}\right\} .
\end{array}
$$

\subsection{LMI Region}

An LMI region is any convex subset $\Phi$ of the complex plane that can be characterized as an LMI in $z$ and $\bar{z}[25]$ as follows:

$$
D=\left\{z \in C: \bar{L}+\bar{M} z+\bar{M}^{T} \bar{z}<0\right\}
$$

for some fixed real matrices $\bar{M}$ and $\bar{L}=\bar{L}^{T}$, where $\bar{z}$ is a complex number. This class of regions encompasses half planes, strips, conic sectors, disks, ellipses, and any intersection of the above. From [25], we find that all eigenvalues of the matrix $A$ are in the LMI region $\left\{z \in C:\left[\bar{l}_{i j}+\bar{m}_{i j} z+\bar{m}_{j i} \bar{z}\right]_{i, j}<0\right\}$ if and only if there exists a symmetric matrix $X$ such that

$$
\left[\bar{l}_{i j} X+\bar{m}_{i j} A^{T} X+\bar{m}_{j i} X A\right]_{i, j}<0, \quad X>0
$$

Also, here we need to include the change of variables and remove the parameter-varying terms, this is done in (3.19). The LMI is obtained in a manner similar to the one that was used for the $\mathscr{H}_{\infty}$ constraint:

$$
\left(\begin{array}{cc}
\Sigma_{4} & \Sigma_{5} \\
* & \Sigma_{3}
\end{array}\right)<0
$$

where

$$
\Sigma_{5}=\left[\begin{array}{llllllll}
\epsilon_{1} P_{1} & N_{1}^{T} & \epsilon_{1} P_{2} & N_{2}^{T} & \epsilon_{2} P_{3} & N_{3}^{T} & \epsilon_{2} P_{4} & N_{4}^{T}
\end{array}\right]
$$

and $\Sigma_{4}$ and the other terms in $\Sigma_{5}$ are defined in the appendix.

Remark 3.2. It is observed that the inequalities (3.14), (3.15), and (3.19) are linear in $(X, Y, \widehat{A}, \widehat{B}, \widehat{C}, \widehat{D})$ and thus the standard LMI techniques can be exploited to find the LMI solutions. It is also seen from the above results that there exists much freedom contained in the design of control law, such as the choices of appropriate $\epsilon_{1}$ and $\epsilon_{2}$. This design freedom can be exploited to achieve other desired closed loop properties. 


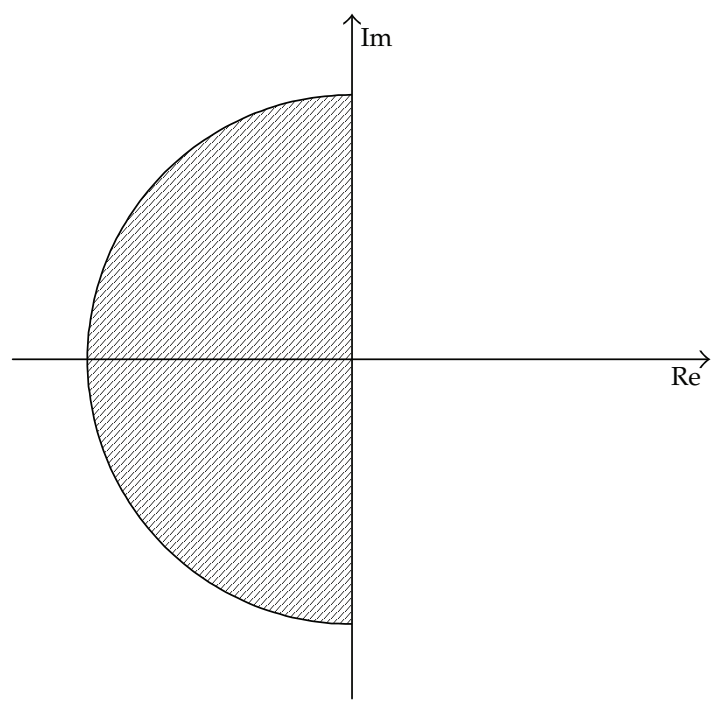

Figure 3: LMI region $\Phi$.

The desired region $\Phi$ is a disk (Figure 3), with center located along the $x$-axis (distance $q$ from the origin) and radius $r$. This determines the region

$$
\Phi=\left(\begin{array}{cc}
-r & q+z \\
q+\bar{z} & -r
\end{array}\right)
$$

From this we can find the matrices $\bar{L}$ and $\bar{M}$, which are the two matrices that determine the LMI region.

All constraints in (3.14), (3.15), and (3.19) are now subjected to the minimization of the objective function, which is the $\mathscr{H}_{\infty}$ norm. They need to be solved in terms of $(X, Y, \widehat{A}, \widehat{B}, \widehat{C}, \widehat{D})$.

Once all these matrices are obtained, the controller matrices are computed in the following way. First we obtain $M$ and $N$ from the factorization problem

$$
M N^{T}=I-X Y .
$$

Second, the controller matrices are computed from the following relationship:

$$
\begin{gathered}
D_{k}=\widehat{D}, \\
C_{k}=\left(\widehat{C}-D_{k} C_{2} X\right)\left(M^{T}\right)^{-1}, \\
B_{k}=N^{-1}\left(\widehat{B}-\Upsilon B_{2 n} D_{k}\right), \\
A_{k}=N^{-1}\left(\widehat{A}-N B_{k} C_{2} X-\Upsilon B_{2 n} C_{k} M^{T}-\Upsilon\left(A_{n}+B_{2 n} D_{k} C_{2}\right) X\right)\left(M^{T}\right)^{-1} .
\end{gathered}
$$




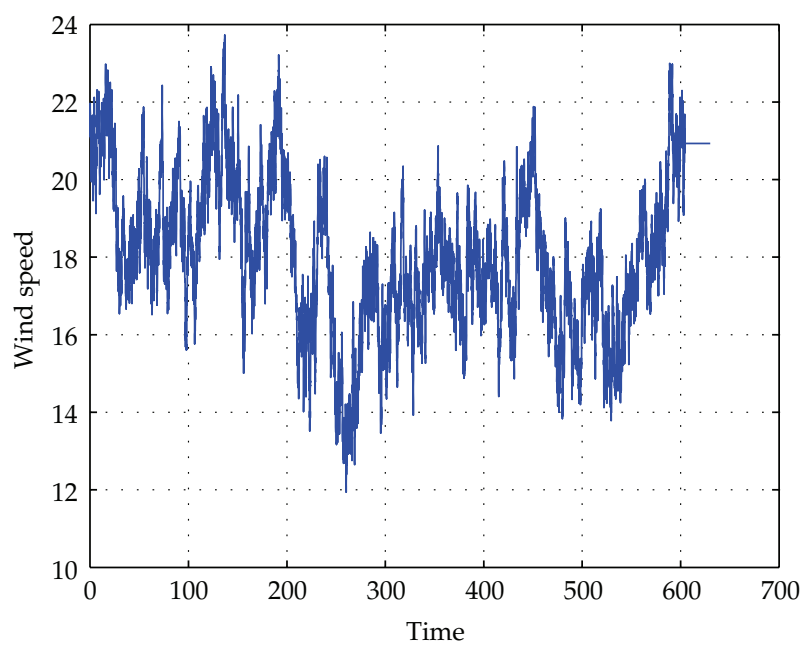

Figure 4: Wind profile.

\section{Simulation Results}

The simulations are carried out with FAST software interfaced with MATLAB/Simulink. The controllers are tested on the fully nonlinear system with 22 out of 24 DOFs enabled. Yaw and platform surge-motion are left out. The wind turbine system is subjected to extreme wind conditions. The wind profile is a 50-year extreme case with an average speed of $18[\mathrm{~m} / \mathrm{s}]$ (Figure 4 ) and a turbulence intensity of $17 \%$. Significant wave hight is $6[\mathrm{~m}]$ with a peak wave period of 10 [s]. The wind profile is obtained from the software Turbsim [26].

Suitable results are found with the following $\mathscr{d}_{\infty}$ performance measure:

$$
z_{\infty}=x_{1}+x_{2}+x_{6}+u \text {. }
$$

The blue line in the plots is the result where the parameter-varying terms are taken into consideration in the controller design. The red line shows the result where the parametervarying terms are left out. We also show NREL's PI gain scheduled controller (cyan colored line) as a reference plot. Our two controllers are designed and tested on exactly the same operating conditions, that is, same performance measure, same pole placement constraint, and same wind condition. From Figures 5 and 6 we see that the blue line is operating more steady around the rated values for the rotor and generator, which are 12.1 [rpm] and 1173.7 $[\mathrm{rpm}]$, respectively. This will in turn result in a smoother torque output, as seen in Figure 7.

Our two controller designs show a large increment in pitching activity, see Figure 8. If we inspect the pitching rate, we see that it is not more than $5-10[\mathrm{deg} / \mathrm{s}]$ and hence should be within the wind turbine's limit. The blue line in Figure 9 shows that the amplitude of the oscillations is lower in the fore-aft direction than in the other two plots. From these plots we see that the results are according to the controller objectives. 


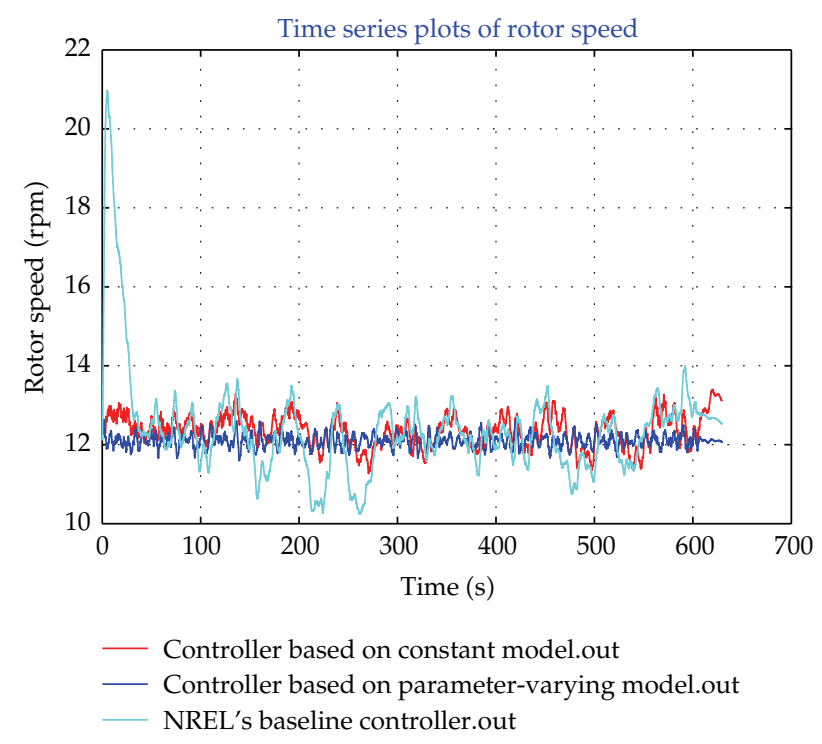

Figure 5: Rotor speed.

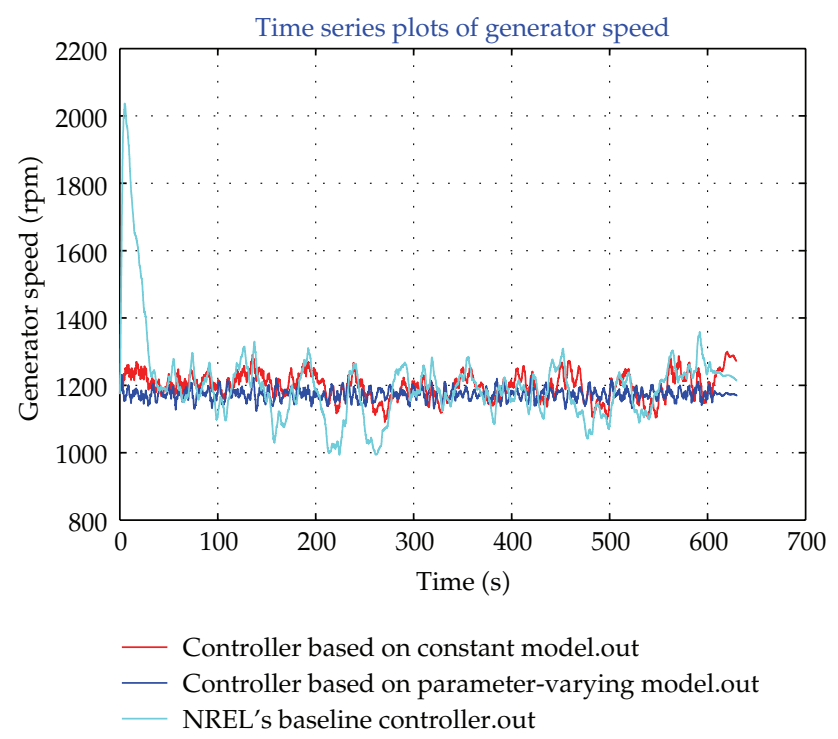

Figure 6: Generator speed.

\section{Conclusions}

In this paper we have obtained and linearized a wind turbine model using the commercial software FAST. The output from the linearization is a family of models describing the turbine system at each 10th azimuth angle. This family of models is converted into one parametervarying model. The new model is dependent on the azimuth angle. In this way we can make the control design based on a model consisting of more information than if we had done it the conventional way, which is to use the average of the family of models. The controller is tested 


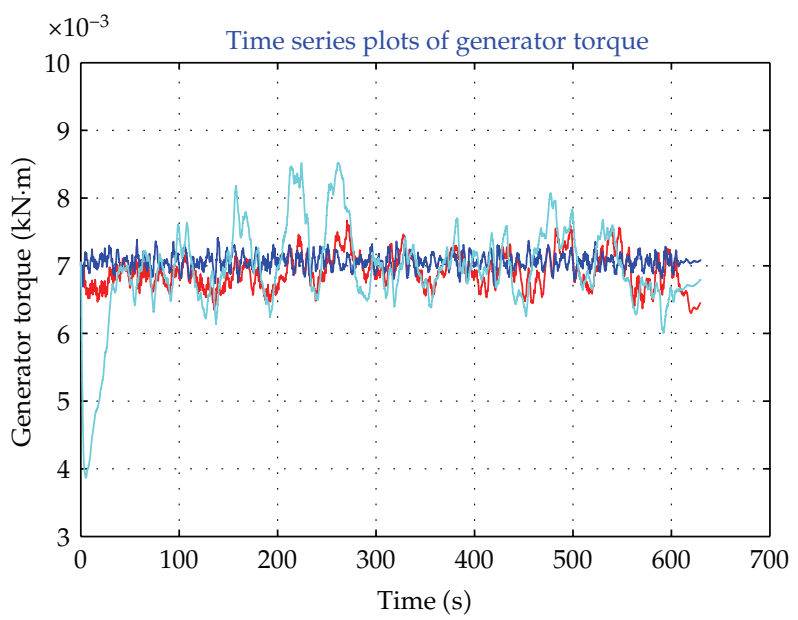

— Controller based on constant model.out
C Controller based on parameter-varying model.out

NREL's baseline controller.out

Figure 7: Generator torque.

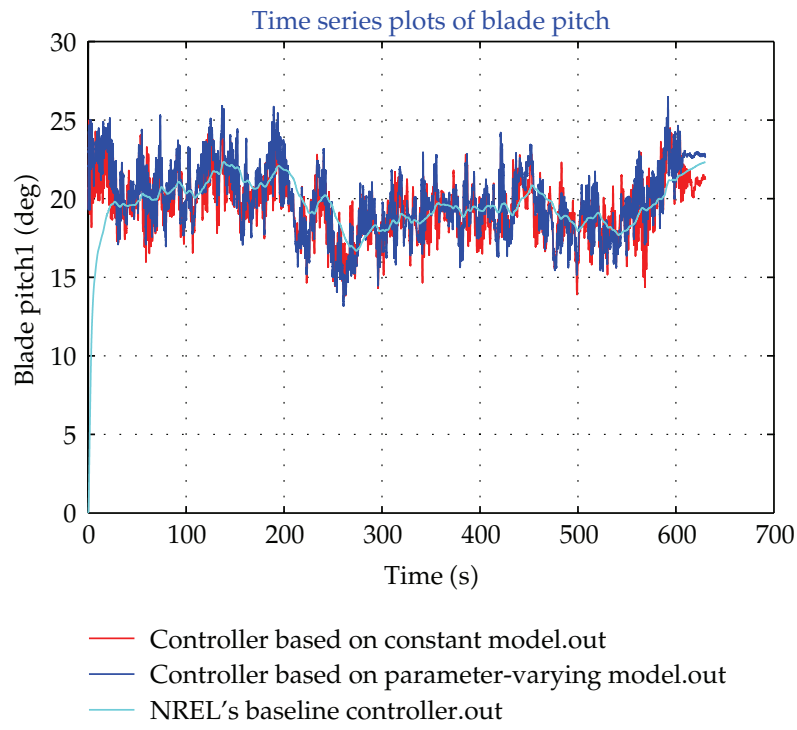

Figure 8: Blade pitch.

on the fully nonlinear system subjected to 50-year extreme wind conditions. The simulation results show a comparison between controller design done with the new method and done the conventional way. The plots show that the simulation results meet our control objectives. follows.

Based on the results in this paper, interesting future research may be prospective as 


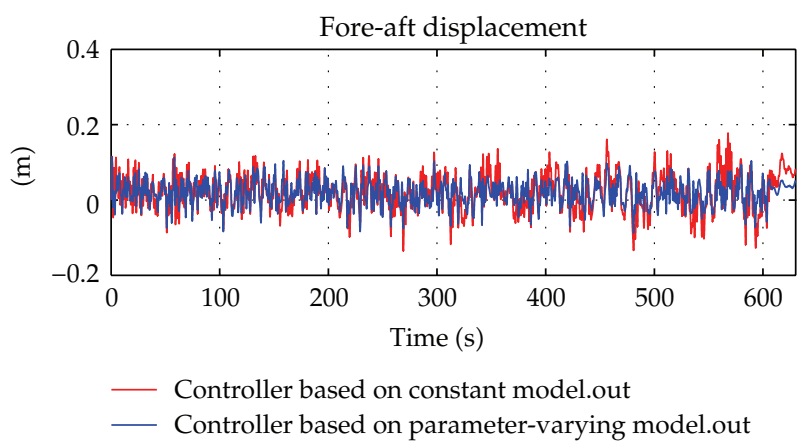

(a)

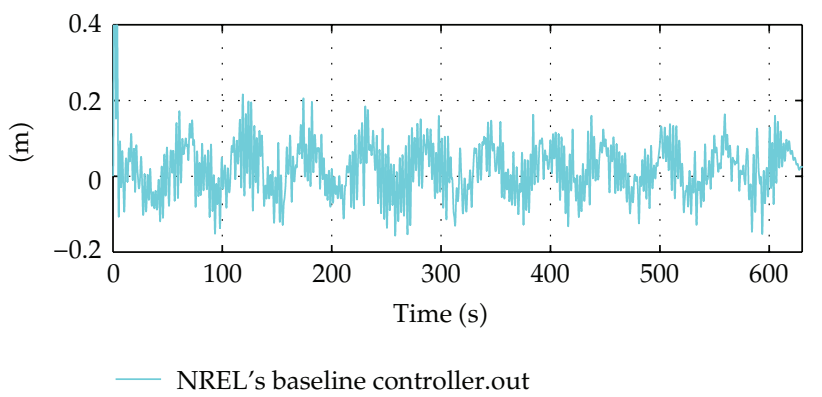

(b)

Figure 9: Tower fore-aft.

(1) It is worth noting that in this paper a constant controller is designed for a parameter-varying model. A next step could be to design a parameter-varying controller, where the scheduling parameter is the azimuth angle.

(2) The methods presented in $[27,28]$ can be used for a stochastic model of a wind turbine system with constrained information exchange and a partial knowledge of the state variables.

(3) Fault detection and control design for wind turbine systems over a network (see, for instance, $[29,30])$ can be studied in the framework of this paper.

(4) Though the addressed issue is the control problem, the methods proposed in the paper can be extended to filtering problems (see, for instance, [31]).

\section{Appendix}

The size of the $A$ matrix is $6 \times 6$, and the $B$ matrix has size $6 \times 1$. Only the last three rows are shown in Figures 10 and 11, respectively. The first three rows contain either constant or zero values. The blue line shows how the 36 linear models are distributed along the 360 


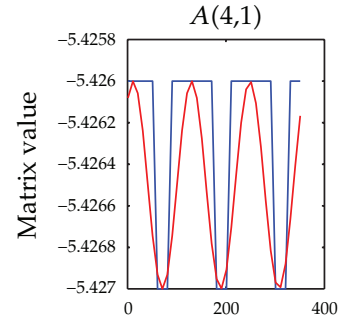

(a)

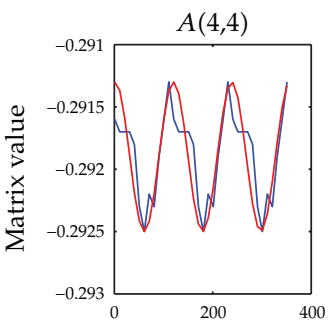

(d)

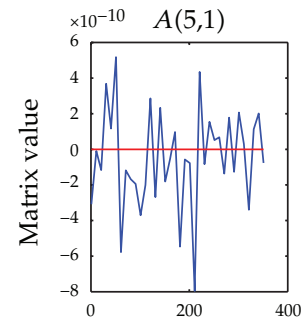

(g)

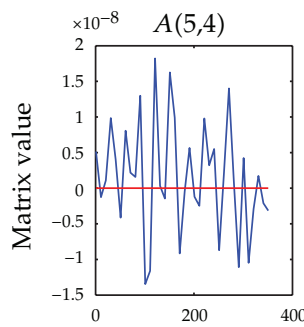

(j)

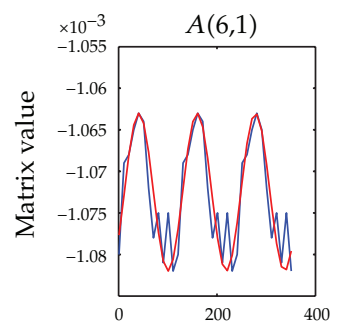

(m)

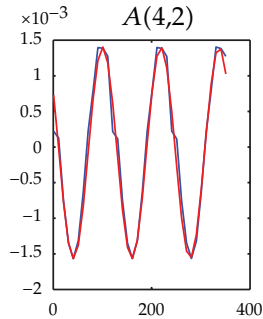

(b)

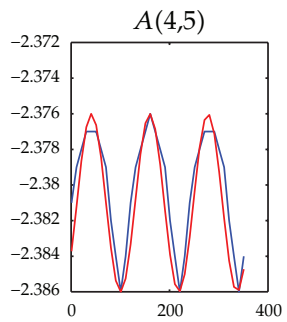

(e)

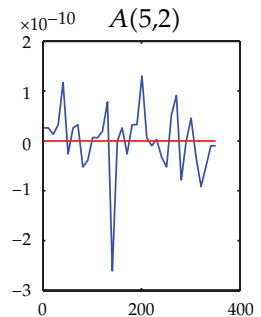

(h)

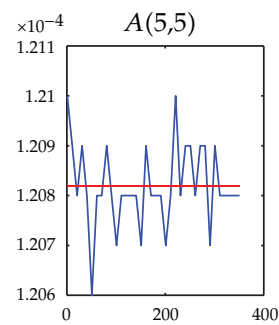

(k)

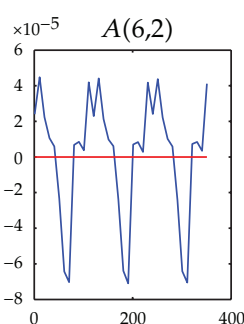

(n)

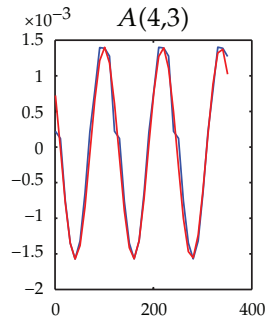

(c)

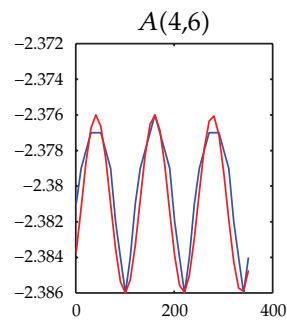

(f)

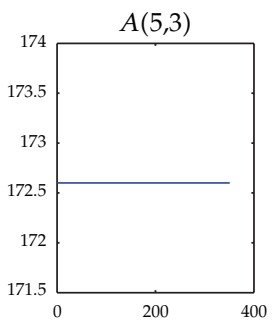

(i)

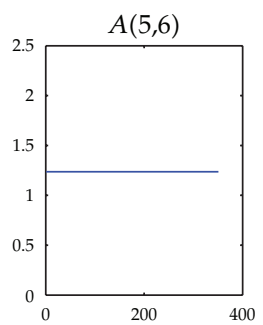

(1)

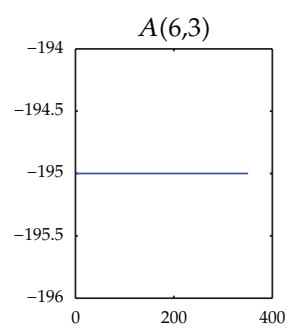

(o)

Figure 10: Continued. 


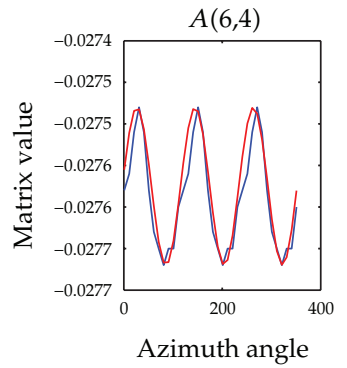

(p)

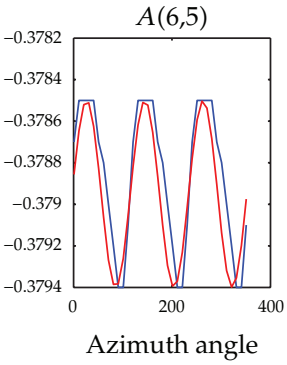

(q)

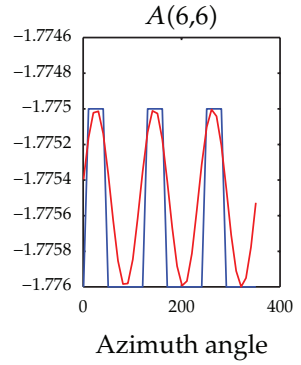

$(\mathrm{r})$

Figure 10: Parameters in A matrix rows 4-6.

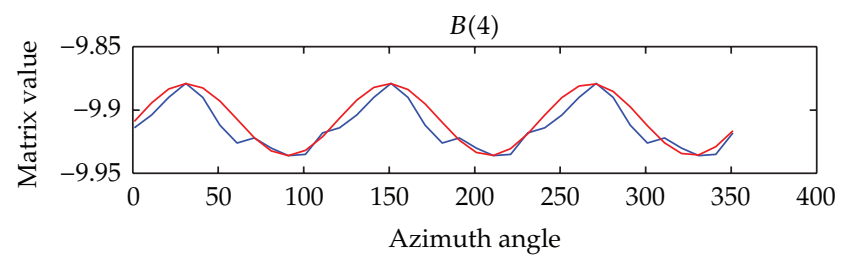

(a)

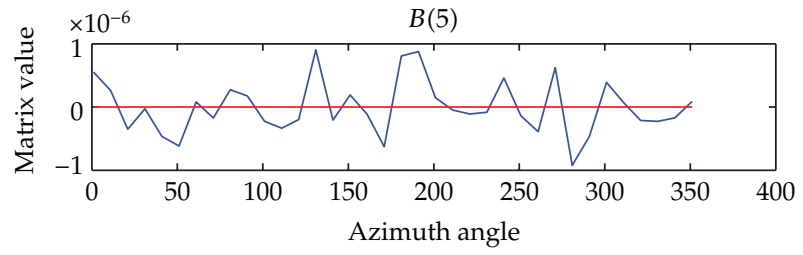

(b)

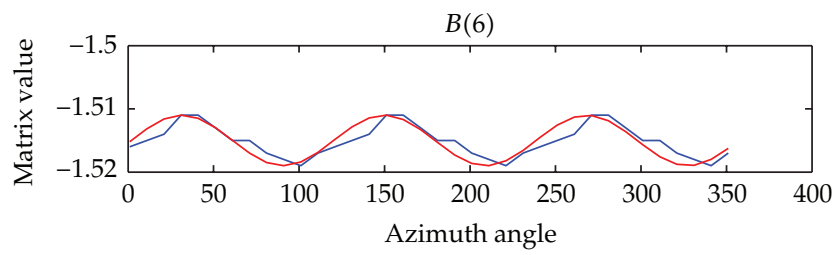

(c)

Figure 11: Parameters in B-matrix rows 4-6.

azimuth angles. The red line shows our attempt to emulate these periodic matrix values with a function on the form $A_{n}+\Delta A(z)$ for the $A$ matrix and $B_{2 n}+\Delta B(z)$ for the $B$ matrix

$$
\Sigma_{1}=\left(\begin{array}{cccc}
\operatorname{sym}\left(A X+B_{2} \widehat{C}\right) & \widehat{A}^{T}+A+B_{2} \widehat{D} C_{2} & B_{1}+B_{2} \widehat{D} D_{21} & X C_{1 i}^{T}+\widehat{C}^{T} D_{21}^{T} \\
* & \operatorname{sym}\left(\Upsilon A+\widehat{B} C_{2}\right) & \gamma B_{1}+\widehat{B} D_{21} & C_{1 i}^{T}+C_{2}^{T} \widehat{D}^{T}+D_{2 i}^{T} \\
* & * & -\gamma I & D_{1 i}^{T}+D_{21}^{T} \widehat{D} D_{2 i}^{T} \\
* & * & * & -\gamma I
\end{array}\right),
$$




$$
\begin{aligned}
& G_{1}=\left[\begin{array}{ll}
F_{1} & 0_{1 \times 8}
\end{array}\right]^{T}, \quad G_{2}=\left[\begin{array}{lll}
0_{1 \times 6} & Y F_{1} & 0_{1 \times 2}
\end{array}\right]^{T}, \\
& G_{3}=\left[\begin{array}{ll}
F_{2} & 0_{1 \times 8}
\end{array}\right]^{T}, \quad G_{4}=\left[\begin{array}{lll}
0_{1 \times 6} & Y F_{2} & 0_{1 \times 2}
\end{array}\right]^{T}, \\
& H_{1}=\left[E_{11 a} X+E_{11 b} \widehat{C} \quad E_{11 a}+E_{11 b} \widehat{D} C_{2} \quad E_{11 b} \widehat{D} D_{21} 0\right] \text {, } \\
& H_{2}=\left[E_{12 a} X+E_{12 b} \widehat{C} \quad E_{12 a}+E_{12 b} \widehat{D} C_{2} \quad E_{12 b} \widehat{D} D_{21} 0\right] \text {, } \\
& H_{3}=\left[\begin{array}{lll}
E_{21 a} X+E_{21 b} \widehat{C} \quad E_{21 a}+E_{21 b} \widehat{D} C_{2} \quad E_{21 b} \widehat{D} D_{21} 0
\end{array}\right] \text {, } \\
& H_{4}=\left[\begin{array}{lll}
E_{22 a} X+E_{22 b} \widehat{C} \quad E_{22 a}+E_{22 b} \widehat{D} C_{2} \quad E_{22 b} \widehat{D} D_{21} 0
\end{array}\right], \\
& \Sigma_{4}=\left(\bar{L} \otimes\left(\begin{array}{cc}
X & I \\
I & Y
\end{array}\right)+\bar{M} \otimes\left(\begin{array}{cc}
A X+B \widehat{C} & A+B \widehat{D} C \\
\widehat{A} & Y A+\widehat{B} C
\end{array}\right)\right. \\
& \left.+\bar{M}^{T} \otimes\left(\begin{array}{cc}
A X+B \widehat{C} & A+B \widehat{D C} C \\
\widehat{A} & \Upsilon A+\widehat{B C}
\end{array}\right)^{T}\right), \\
& G_{1}=\left[\begin{array}{ll}
F_{1} & 0_{1 \times 6}
\end{array}\right]^{T}, \quad G_{2}=\left[\begin{array}{lll}
0_{1 \times 6} & Y F_{1}
\end{array}\right]^{T}, \quad G_{3}=\left[\begin{array}{ll}
F_{2} & 0_{1 \times 6}
\end{array}\right]^{T}, \quad G_{4}=\left[\begin{array}{ll}
0_{1 \times 6} & Y F_{2}
\end{array}\right]^{T}, \\
& H_{1-2}=\left[\begin{array}{lll}
E_{11 a} X+E_{11 b} \widehat{C} & E_{11 a}+E_{11 b} \widehat{D} C_{2}
\end{array}\right], \quad H_{2-2}=\left[\begin{array}{lll}
E_{12 a} X+E_{12 b} \widehat{C} & E_{12 a}+E_{12 b} \widehat{D} C_{2}
\end{array}\right], \\
& H_{3-2}=\left[\begin{array}{lll}
E_{21 a} X+E_{21 b} \widehat{C} & E_{21 a}+E_{21 b} \widehat{D} C_{2}
\end{array}\right], \quad H_{4-2}=\left[\begin{array}{lll}
E_{22 a} X+E_{22 b} \widehat{C} & E_{22 a}+E_{22 b} \widehat{D} C_{2}
\end{array}\right], \\
& N_{1}=I_{2 \times 2} \otimes H_{1-2}, \quad N_{2}=I_{2 \times 2} \otimes H_{2-2}, \quad N_{3}=I_{2 \times 2} \otimes H_{3-2}, \quad N_{4}=I_{2 \times 2} \otimes H_{4-2}, \\
& H_{1}=M \otimes G_{1-2}, \quad H_{2}=M \otimes G_{2-2}, \quad H_{3}=M \otimes G_{3-2}, \quad H_{4}=M \otimes G_{4-2} .
\end{aligned}
$$

\section{Acknowledgments}

This work has been (partially) funded by Norwegian Centre for Offshore Wind Energy (NORCOWE) under Grant 193821/S60 from Research Council of Norway (RCN). NORCOWE is a consortium with partners from industry and science, hosted by Christian Michelsen Research.

\section{References}

[1] EWEA, “Wind Industry Factsheet,” Tech. Rep., European Wind Energy Association (EWEA), 2004.

[2] Y. D. Song, B. Dhinakaran, and X. Y. Bao, "Variable speed control of wind turbines using nonlinear and adaptive algorithms," Journal of Wind Engineering and Industrial Aerodynamics, vol. 85, no. 3, pp. 293-308, 2000.

[3] R. Melcioa, V. M. F. Mendesc, and J. P. S. Cataloa, "Comparative study of power converter topologies and control strategies for the harmonic performance of variable-speed wind turbine generator systems," Energy, vol. 36, no. 1, pp. 520-529, 2011.

[4] A. Kusiak, W. Li, and Z. Song, "Dynamic control of wind turbines," Renewable Energy, vol. 35, no. 2, pp. 456-463, 2010.

[5] A. Kusiak and Z. Zhang, "Control of wind turbine power and vibration with a data-driven approach," Renewable Energy, vol. 43, pp. 73-82, 2012. 
[6] R. Eide and H. R. Karimi, "Control design methodologies for vibration mitigation on wind turbine systems," in Vibration Control, F. Beltran-carbajal, Ed., pp. 217-242, INTECH.

[7] H. R. Karimi and T. Bakka, "Stochastic Analysis and $H_{\infty}$ Control ofWind Turbine Systems with Wireless Sensor Networks," in Proceedings of the 24th International Congress on Condition Monitoring and Diagnostics Engineering Management, pp. 1086-1095, 2011.

[8] H. R. Karimi, "Wavelet-based optimal control of a wind turbine system: a computational approach," Journal of Advanced Mechanical Design, Systems, and Manufacturing, vol. 5, no. 3, pp. 171-186, 2011.

[9] F. D. Bianchi, H. de Battista, and R. J. Mantz, Wind Turbine Control Systems, Springer, 2007.

[10] C. W. Taylor, F. R. Nassief, and R. L. Cresap, "Northwest power pool transient stability and load shedding controls for generation-load imbalances," IEEE transactions on power apparatus and systems, vol. 100, no. 7, pp. 3486-3495, 1981.

[11] A. Mullane, G. Lightbody, and R. Ya, "Adaptive control of variable speed wind turbines," Revue Des Energies Renouveables: Power Engineering, pp. 101-110, 2001.

[12] F. Valenciaga, P. F. Puleston, P. E. Battaiotto, and R. J. Mantz, "An adaptive feedback linearization strategy for variable speed wind energy conversion systems," International Journal of Energy Research, vol. 24, no. 2, pp. 151-161, 2000.

[13] M. Steinbuch and O. H. Bosqra, "Optimal output feedback of a wind energy conversion system," in Proceedings of the Power Systems Modelling and Control Applications, A. J. Calvaer, Ed., IFAC Proceeding series, pp. 313-319, September 1988.

[14] R. Rocha, L. S. Martins Filho, and M. V. Bortolus, "Optimal multivariable control for wind energy conversion system - A comparison between $\mathrm{H}_{2}$ and $\mathrm{H}_{\infty}$ controllers," in Proceedings of the 44th IEEE Conference on Decision and Control, and the European Control Conference, (CDC-ECC'05), pp. 7906-7911, December 2005.

[15] T. Bakka, H. R. Karimi, and N. A. Duffie, "Gain scheduling for output $\mathscr{l}_{\infty}$ control of offshore wind turbine," in Proceedings of the 22nd International Offshore and Polar Engineering Conference, pp. 496-501, Rhodes, Greece, June 2012.

[16] T. Bakka and H. R. Karimi, "Robust output feedback $\mathscr{\ell}_{\infty}$ control synthesis with pole placement for offshore wind turbine system: an LMI approach," in IEEE International Conference on Control Applications (CCA '12), Dubrovnik, Croatia, October 2012.

[17] T. Bakka and H. R. Karimi, "Mixed $\mathrm{H}_{2} / \mathrm{H}_{\infty}$ control design for wind turbine system with pole placement constraints," in Proceedings of the 31st Chinese Control Conference, pp. 4775-4780, Hefei, China, July 2012.

[18] T. J. Larsen, “How 2 HAWC2, the users manual,” Ris-R-1597(ver. 3-9)(EN), 2009.

[19] J. Jonkman and M. L. Buhl Jr., "FAST Users Guide," Tech. Rep. NREL/EL-500-38230, National Renewable Energy Laboratory, 2005.

[20] C. L. Bottasso and A. Croce, Cp-Lambda User Manual, Dipartimento di Ingnegneria Aerospaziale, Politecnico di Milano, Milano, Italy, 2009.

[21] J. Jonkman, S. Butterfield, W. Musial, and G. Scott, “Definition of a 5-MW referencewind turbine for offshore system development," Tech. Rep. NREL/TP-500-38060, National Renewable Energy Laboratory, 2009.

[22] J. Lfberg, "YALMIP: a toolbox for modeling and optimization in MATLAB," in Proceedings of the IEEE International Symposium on Computer Aided Control Systems Design, (CACSD '04), pp. 284-289, Taipei, Taiwan, 2004.

[23] C. Scherer, P. Gahinet, and M. Chilali, "Multiobjective output-feedback control via LMI optimization," IEEE Transactions on Automatic Control, vol. 42, no. 7, pp. 896-911, 1997.

[24] P. P. Khargonekar, I. R. Petersen, and K. Zhou, "Robust stabilization of uncertain linear systems: quadratic stabilizability and Hoo control theory," IEEE Transactions on Automatic Control, vol. 35, no. 3, pp. 356-361, 1990.

[25] M. Chilali and P. Gahinet, " $H_{\infty}$ design with pole placement constraints: an LMI approach," IEEE Transactions on Automatic Control, vol. 41, no. 3, pp. 358-367, 1996.

[26] B. J. Jonkman, “TurbSim User's Guide: Version 1.50,” Tech. Rep. NREL/EL-500-46198, National Renewable Energy Laboratory, 2009.

[27] Z. Wang, D. W. C. Ho, H. Dong, and H. Gao, "Robust $H_{\infty}$ finite-horizon control for a class of stochastic nonlinear time-varying systems subject to sensor and actuator saturations," IEEE Transactions on Automatic Control, vol. 55, no. 7, pp. 1716-1722, 2010.

[28] H. Dong, Z. Wang, D. W. C. Ho, and H. Gao, "Robust $H_{\infty}$ fuzzy output-feedback control with multiple probabilistic delays and multiple missing measurements," IEEE Transactions on Fuzzy Systems, vol. 18, no. 4, pp. 712-725, 2010. 
[29] H. Dong, Z. Wang, and H. Gao, "Robust $H_{\infty}$ filtering for a class of nonlinear networked systems with multiple stochastic communication delays and packet dropouts," IEEE Transactions on Signal Processing, vol. 58, no. 4, pp. 1957-1966, 2010.

[30] B. Shen, Z. Wang, H. Shu, and G. Wei, "Robust $H_{\infty}$ finite-horizon filtering with randomly occurred nonlinearities and quantization effects," Automatica, vol. 46, no. 11, pp. 1743-1751, 2010.

[31] B. Shen, Z. Wang, H. Shu, and G. Wei, " $H_{\infty}$ filtering for nonlinear discrete-time stochastic systems with randomly varying sensor delays," Automatica, vol. 45, no. 4, pp. 1032-1037, 2009. 


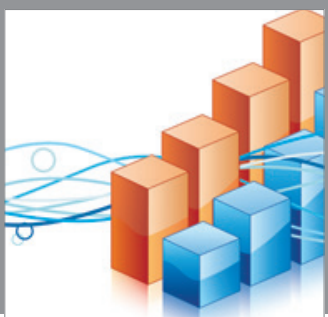

Advances in

Operations Research

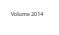

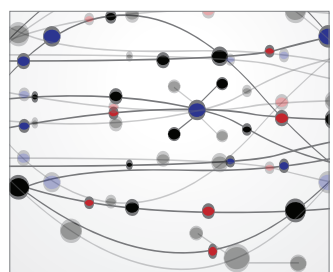

\section{The Scientific} World Journal
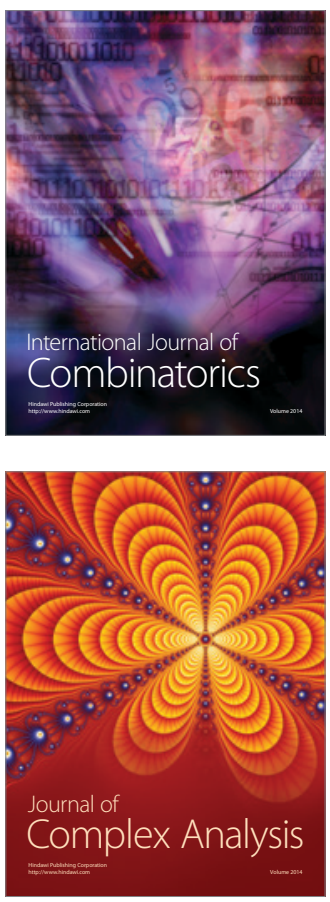

International Journal of

Mathematics and

Mathematical

Sciences
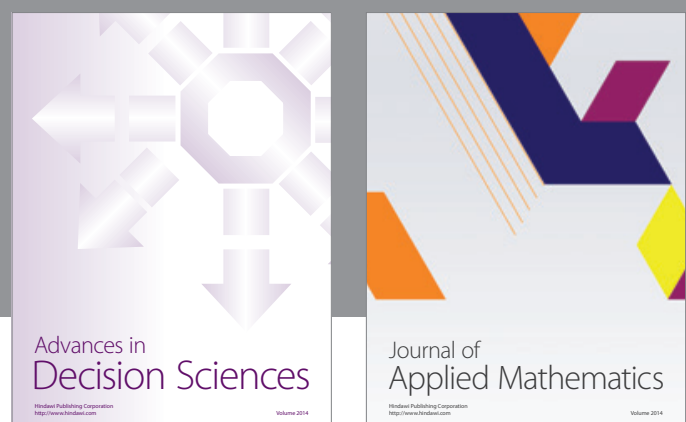

Journal of

Applied Mathematics
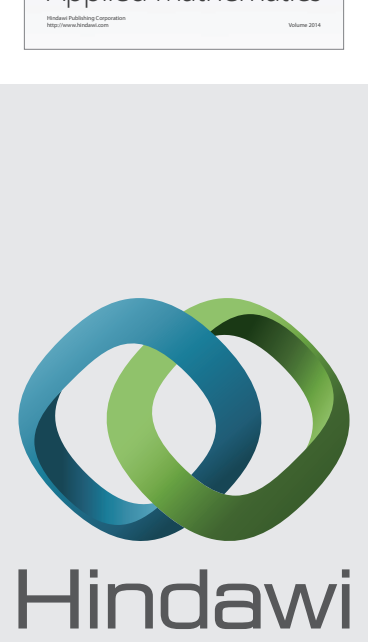

Submit your manuscripts at http://www.hindawi.com
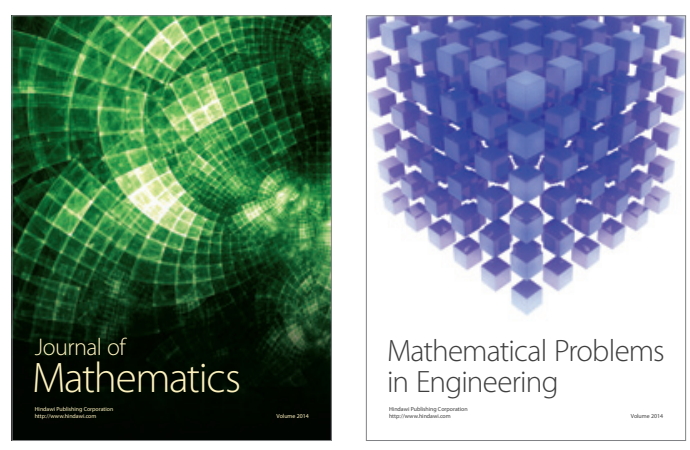

Mathematical Problems in Engineering
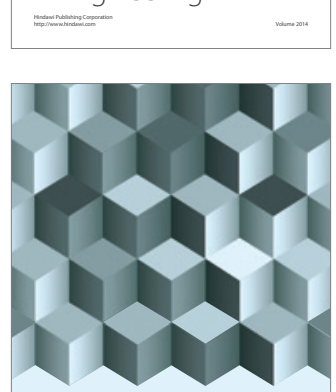

Journal of

Function Spaces
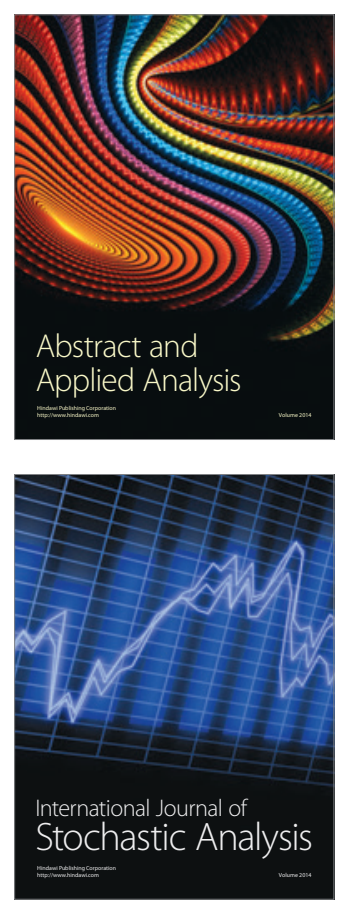

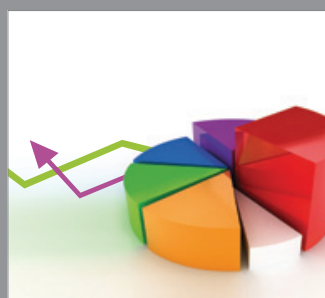

ournal of

Probability and Statistics

Promensencen
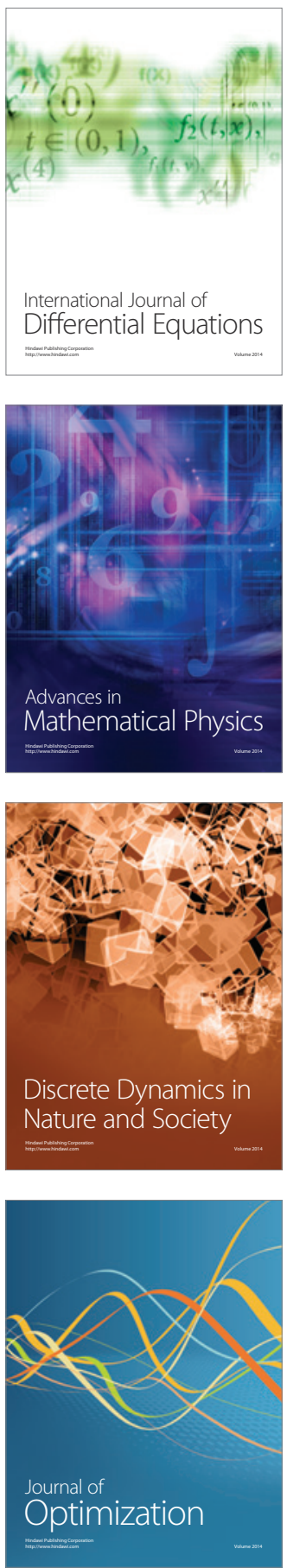